\title{
Endovascular management of suprascapular artery pseudoaneurysm
}

\author{
Scott Prater, MD, Daniel A. Marichal, MD, and Chet Rees, MD
}

Pseudoaneurysms can be debilitating for patients and occasionally life threatening. Many imaging modalities may be used to assess and define these vascular lesions, and various methods have been employed in their treatment. Our patient suffered trauma to his left shoulder resulting in fracture of his clavicle following an automobile accident and subsequently was found to have a pseudoaneurysm of his left suprascapular artery on imaging. This was treated by transarterial embolization, which resulted in immediate relief of his symptoms. Endoluminal management offers a safe and effective way of treating these lesions.

$\mathrm{n}$ an automobile accident in 2007, a 40-year-old man fractured his left clavicle. Although the fracture was repaired, he continued to have pain in the left supraclavicular region that radiated to the left lateral and posterior aspects of his neck. He also reported feeling a mass just beneath his clavicle. In July 2009, the symptoms worsened when he lifted heavy objects. After physical examination by the referring orthopedic surgeon, he was imaged with contrast-enhanced computed tomography (CT) (Figure 1). He also had noncontrast magnetic resonance (MR) imaging, which demonstrated only a partial-thickness articular surface rotator cuff tear and magnetic susceptibility artifact (not shown).

The patient was referred to the vascular and interventional radiology department at Baylor University Medical Center, where he underwent diagnostic catheter angiography in the digital fluoroscopy suite (Siemens Artis). The angiogram

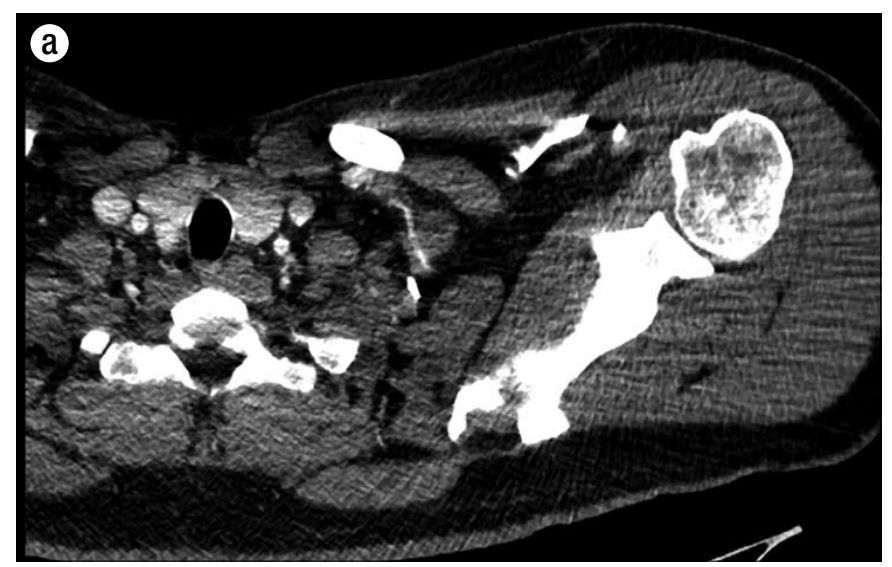

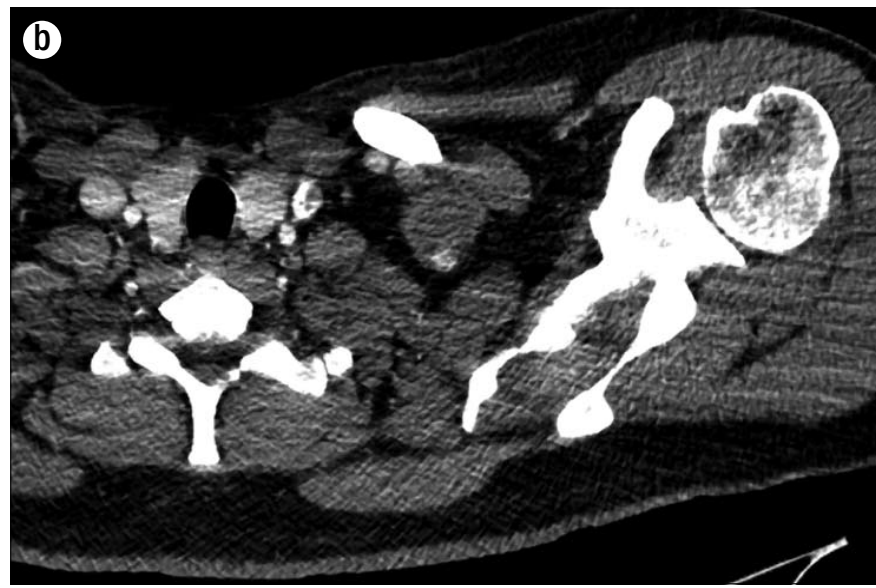

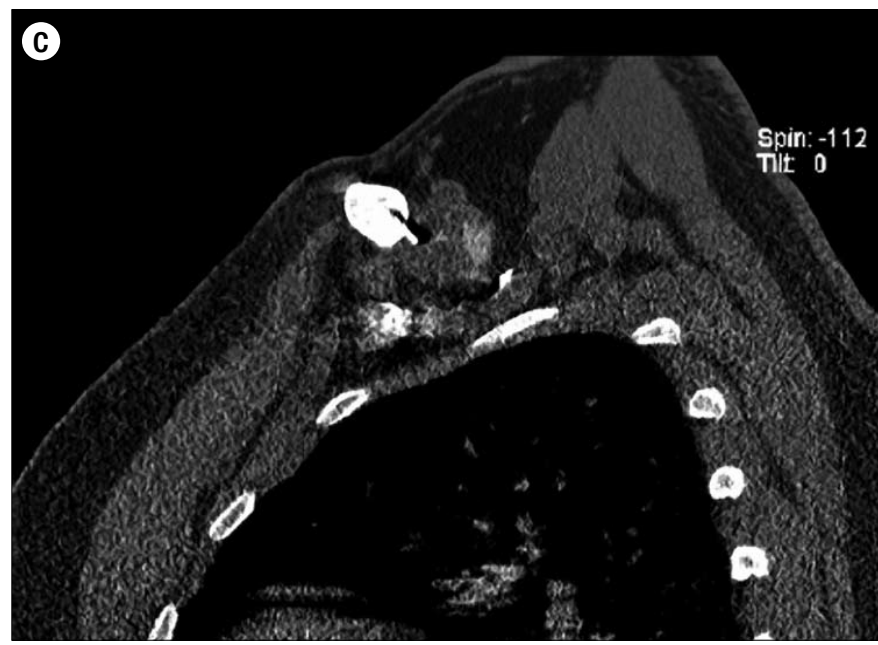

Figure 1. CT scans in July 2009. (a, b) Axial views through the left shoulder demonstrate contrast extending into a saccular outpouching posterior and superior to the clavicle consistent with left suprascapular artery pseudoaneurysm. (c) Sagittal reformatted image through the supraclavicular region demonstrates retained contrast within the pseudoaneurysm.

demonstrated a 21-mm pseudoaneurysm arising from the suprascapular artery (Figure 2).

From the Department of Radiology, Baylor University Medical Center, Dallas, Texas.

Corresponding author: Scott Prater, MD, Department of Radiology, Baylor University Medical Center, 3500 Gaston Avenue, Dallas, Texas 75246 (e-mail: Scott.Prater@BaylorHealth.edu). 


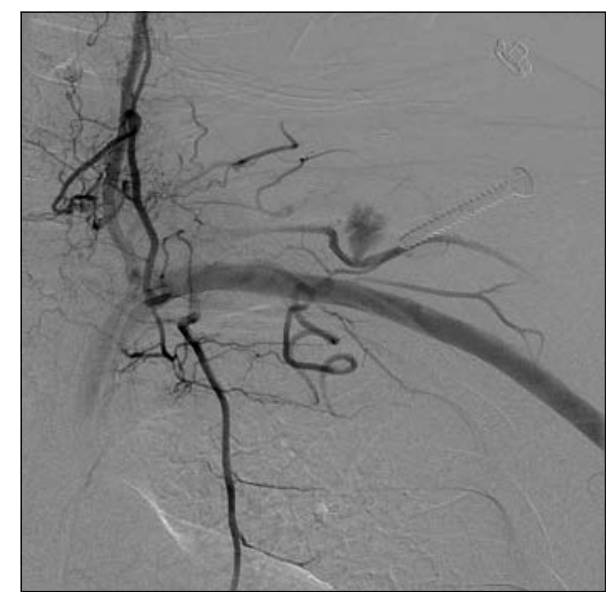

Figure 2. Digital subtraction angiogram selecting the of contrast is demonstrated extending off the suprascapular artery, compatible with the pseudoaneurysm ing the lesion.

of interest. The surgical screw was from prior surgical repair.

After discussing the findings with the patient and obtaining informed consent, definitive treatment was planned. The left subclavian artery was catheterized with a $5 \mathrm{~F}$ vertebral catheter (Merit Medical, South Jordan, Utah). Subsequently, the left suprascapular artery was selectively catheterized by a coaxial technique with a 3F microcatheter (Progreat, Terumo Medical Corp., Somerset, NJ). Complex helical 0.018-inch fibered platinum microcoils (Boston Scientific, Natick, MA) were subsequently placed distal to the aneurysm origin (Figure 3). Coils were then placed proximal and within the origin of the pseudoaneurysm (Figure 4). The patient tolerated the procedure well and reported immediate pain relief.

\section{DISCUSSION}

Pseudoaneurysms are the uncommon result of vascular injury. They occur when one or two arterial walls of the artery are dissected, so that a collection of blood seeps through the dissection, maintaining continuity with the arterial lumen. Pseudoaneurysms may rupture, leading to massive hemorrhage and in some cases death. Intraluminal thrombus may occur, incurring ischemic effects such as claudication, ischemia, and even potential loss of limb. Less severe but still debilitating complications such as constant pain and paraesthesias may occur as well. Early detection and diagnosis can prevent the morbidity and death from pseudoaneurysms (1-5).

Pseudoaneurysms have various etiologies, most commonly trauma or iatrogenic injury after endovascular procedures. Other causes include tumor invasion, infection, inflammatory disorders including vasculitis, and penetrating atherosclerotic ulcers $(2,4)$.

Our patient suffered a fracture to his left clavicle after an automobile collision. Fractures to the clavicle are common in blunt-force trauma, representing approximately $2 \%$ to $5 \%$ of all fractures and $44 \%$ of those in the shoulder girdle. Reports of delayed neurovascular complications associated with clavicular fractures are described in the literature, including formation of pseudoaneurysms, which can go unrecognized for years $(2,4$, 5). This appeared to be the case for our patient.

Several imaging modalities can be used to identify pseudoaneurysms. The best noninvasive modalities include Duplex sonography, CT angiography, and MR angiography. Duplex ultrasound has been shown to have a 94\% sensitivity and 97\% specificity for identification of pseudoaneurysms that reside superficially; however, spatial resolution is limited with deeper structures, and ultrasound is less accurate in demonstrating whether a feeding vessel is associated with the pseudoaneurysm in question. The typical appearance of a pseudoaneurysm on ultrasound is a "to-and-fro" pattern, documenting the turbulent flow within the lesion (6-9). Multislice CT angiography has a sensitivity and specificity of $95.1 \%$ and $98.7 \%$, respectively, in detecting pseudoaneurysms in the proximal extremities. The ability to reconstruct the images into a three-dimensional format allows additional information to be obtained, which is useful in treatment planning. In addition, CT offers the ability to detect surrounding extravascular injuries and/or disease entities, as well as provide bony landmarks in surgical planning (3).

Newer imaging modalities such as MR angiography have come into play over the past few years. Some of its advantages include better tissue characterization of surrounding extravascular structures, less image degradation with newer vascular treatment devices (coils, clips, and stents), and the avoidance of ionizing radiation. At times, patients are unable to receive a contrast exam because of allergy or renal insufficiency. MR time of flight imaging allows interrogation of the vasculature without subjecting patients to intravenous iodinated contrast. Shortcomings of MR angiography include decreased detection of surrounding bony anatomy and aerated structures, longer duration of the exam, cost, and reduced availability (10).

Catheter-directed angiography, although invasive, remains the gold standard for identification of a pseudoaneurysm. Angiography can image vascular lesions in real time and gives a clear 
depiction for identifying collateral vessels, allowing assessment of whether the artery supplying flow to the pseudoaneurysm may be permanently excluded (3).

Before the 1990s, the only treatment available for pseudoaneurysms was surgery. Although effective, surgical treatment offers greater risk with higher morbidity. Additional complications include anesthesia, bleeding, and increased risk of infection. Longer recovery time adds additional cost to the patient as well as the health care system (3). Newer modalities have arisen, including sonographic compression. This has been superseded by sonographic thrombin injection, often the treatment of choice for femoral artery pseudoaneurysms, which are usually developed from prior endovascular procedures. Success rates using this technique vary from $91 \%$ to $100 \%$ (8).

More modern techniques in treating pseudoaneurysms have arisen with endoluminal management, which serves to separate the pseudoaneurysm from its circulation. This offers lower cost to the patient with reduced recovery time. With its less-invasive techniques and moderate sedation, the endovascular approach also provides decreased risk and operative morbidity to the patient (2). These techniques include stent-graft placements, coils, and newer modalities such as polymerizing agents including glue $(3,11-13)$. The latter has proven very effective in correcting endoleaks after endovascular aortic aneurysm repair and in the treatment of visceral artery aneurysms (13).

In our case we used platinum coils to embolize the areas proximal and distal to the pseudoaneurysm to completely exclude it from the circulation and prevent backflow from collateral vessels. The patient reported immediate relief, citing cessation of radiating pain as well as a marked decrease in pain overlying the supraclavicular region. He also reported that he no longer felt a mass below his clavicle. A 2-month follow up revealed persistent decreased pain.
1. Sueyoshi E, Sakamoto I, Nakashima K, Minami K, Hayashi K. Visceral and peripheral arterial pseudoaneurysms. AJR Am J Roentgenol 2005;185(3):741-749.

2. Nowlis E, Bilello JF, Venugopal C, Rich NM. Interventional radiographic embolization of a traumatic pseudoaneurysm and associated arteriovenous fistulas 33 years after injury in a Vietnam combat veteran: worth the wait? J Trauma 2009 Aug 21 [Epub ahead of print].

3. Saad NE, Saad WE, Davies MG, Waldman DL, Fultz PJ, Rubens DJ. Pseudoaneurysms and the role of minimally invasive techniques in their management. Radiographics 2005;25(Suppl 1):S173-S189.

4. Heyn J, Ozimek A, Sadeghi-Azandaryani M, Bürklein D, Steckmeier B. Thoracic outlet syndrome caused by a pseudoaneurysm after pseudarthrosis of the clavicle. Eur J Trauma Emerg Surg 2008;34(5):511-514.

5. Kitsis CK, Marino AJ, Krikler SJ, Birch R. Late complications following clavicular fractures and their operative management. Injury 2003;34(1):69-74.

6. Ertzner TW, Powers TA. Pseudoaneurysm detection with Tc-99m-labeled red blood cells. Radiology 1985;154(2):507-512.

7. Coughlin BF, Paushter DM. Peripheral pseudoaneurysms: evaluation with duplex US. Radiology 1988;168(2):339-342.

8. Miranda FC, Garcia RG, Filho CLM, Mendes GF, de Andrade JR, Maurano A, Neto MJF, Funari MBG. Doppler ultrasound-guided percutaneous thrombin injection for treating femoral pseudoaneurysms. Einstein 2008;6(4):428-433.

9. Huda W, Sloan R. Review of Radiologic Physics. Baltimore: Lippincott Williams \& Wilkins, 2003:183.

10. Bowen BC. MR angiography versus $\mathrm{CT}$ angiography in the evaluation of neurovascular disease. Radiology 2007;245(2):357-360.

11. Gulati GS, Gulati MS, Makharia G, Hatimota P, Saikia N, Paul SB, Acharya S. Percutaneous glue embolization of a visceral artery pseudoaneurysm in a case of sickle cell anemia. Cardiovasc Intervent Radiol 2006;29(4):665-668.

12. Zelenák K, Sopilko I, Svihra J, Kliment J. Successful embolization of a renal artery pseudoaneurysm with arteriovenous fistula and extravasations using Onyx after partial nephrectomy for renal cell carcinoma. Cardiovasc Intervent Radiol 2009;32(1):163-165.

13. Tulsyan N, Kashyap VS, Greenberg RK, Sarac TP, Clair DG, Pierce G, Ouriel K. The endovascular management of visceral artery aneurysms and pseudoaneurysms. J Vasc Surg 2007;45(2):276-283. 